\title{
Acute Brucellosis: Presentation and complications in Adults
}

\author{
Mohammad Ahmed Saraya \\ Department of Tropical Medicine, Faculty of Medicine, Zagazig University, Egypt .
}

Corresponding Author Mohammad Ahmed Saraya

Mobile:

+96555499517

E mail:

mdsaraya@yahoo.com

Key words: Acute brucellosis, Presentation, Laboratory findings, Complications
Background and study aim: Brucellosis is one of the important bacterial zoonotic infectious diseases. The epidemiological zone of this infection includes the Arabian Peninsula. This study was conducted to describe the clinical presentation, laboratory findings and frequency of complications in adult brucellosis patients admitted in the hospital

Patients and Methods: This was a retrospective study conducted at the Infectious Diseases Hospital from May 2015 to May 2017. The patients included in this study were diagnosed as acute brucellosis. Diagnosis of acute brucellosis based on epidemiologic data, clinical manifestations, and laboratory investigations. Patients were treated according to the standard guidelines for the management of brucellosis and its complications.
Results: A total 192 patients were enrolled into the study. The main presenting symptoms were Fever (91.6\%), night sweats $(85.9 \%)$, arthralgia (76\%), and low back pain $(63.5 \%)$. The most common focal involvements observed were hepatic involvement (28.1\%), Osteoarticular involvement $(22.4 \%)$, Hematological involvement $(17.1 \%)$ and orchiepididymitis involvement $(9.4 \%)$.

Conclusion: Brucellosis considered an important medical problem. Diagnosis of the disease is not difficult but the diagnosis of its localized forms can occasionally be difficult because of misdiagnosis with other diseases. In humans, Eradication of the disease can be done by the public health education and the control of the disease in animals.

\section{INTRODUCTION}

Brucellosis is one of the important bacterial zoonotic infectious diseases caused by gram negative bacteria, brucella spp. It is a widespread disease with worldwide prevalence and more than 500000 new cases annually [1]. The epidemiological zone of this infection includes Mediterranean basin, the Arabian Peninsula, Indochina, some parts of central Asia and South America [2].

The brucellosis can be transmitted to human by consumption of infected meat from infected domestic animals e.g cattle, goat, sheep, camel, pigs... ect or close contact with the secretions of these infected animals [3]. The transmission also possible occurred by consumption of unpasteurized dairy such as ice cream, soft cheese, butter but yogurt and hard cheese have a lower risk of transmission than others due to lactic fermentation [4].
The forms of the clinical course of brucellosis in humans are acute, subacute and chronic. Patients were classified into three groups according to their history, clinical picture and the time of clinical presentation: acute (0-2 months), sub-acute (3-12 months) and chronic (>1 year) [5].

This study was conducted to describe the clinical presentation, laboratory findings and frequency of complications in adult brucellosis patients admitted in the hospital.

\section{PATIENTS AND METHODS}

This is a descriptive study conducted from May 2015 to May 2017at the Infectious Diseases Hospital (IDH), Kuwait. The patients included in this study were diagnosed as acute brucellosis. Diagnosis of acute brucellosis was clinically based on the history of contact with animals or consumption of unpasteurized dairy 
and also, based on the presence of symptoms, e.g. fever, chills, night sweats, myalgia, anorexia, headache, joint pain and confirmed serologically by positive of brucella Immunoglobulin M (IgM) antibodies, standard tube agglutination titer (SAT) $\geq 1: 160$ or isolation of a Brucella spp. from blood cultures.

All patients were subjected to history taking and thorough clinical examination; tests for liver function test (LFT), kidney profile (KP), complete blood count $(\mathrm{CBC})$, malaria film, erythrocyte sedimentation rate, blood glucose, rheumatoid factor (RF), C-reactive protein (CRP), Coagulation profiles, urine analysis and chest radiograph were performed on the day of admission and during stay in the hospital.

The patients were called for outpatient follow up after discharge from the hospital. The outpatient visits were every two weeks until full recovery. At each visit, LFT, KP, CBC, and CRP were investigated.

Regarding complicated brucellosis, diagnosis of osteoarticular involvement e.g arthritis, sacroiliitis, spondylitis, were determined by appropriate findings on physical examination and radiological investigations e.g X-ray and magnetic resonance imaging (MRI) if indicated. Diagnosis of genitorurinary system involvement e.g orchitis and/or epididymitis was defined by finding tenderness and swelling of scrotum, testis and epididymis and confirmed by ultra-sonography.

Diagnosis of Hepatic involvement was defined as the presence of more than five-fold of normal levels of alanine aminotransferase (ALT) and aspartate aminotransferase (AST). Diagnosis of brucella endocarditis was determined by cardiac murmur and confirmed by detection of vegetation using transthoracic echocardiography study. Diagnosis of neurobrucellosis was defined by neurological manifestations and confirmed by positivity of SAT and/or positivity of cerebrospinal fluid (CSF) culture and abnormal findings in cerebrospinal fluid analyses (protein $>45 \mathrm{mg} / \mathrm{dL}$, glucose $<2: 3$ of the blood glucose level, $>10$ leukocyte/mm3).

Management was done as per standard guidelines for the management of brucellosis and its complications. The regimens included different combinations of antibiotics e.g oral doxycycline (100 mg/12h), oral rifampin (600 or $900 \mathrm{mg} / 24 \mathrm{~h}$ ), gentamicin infusion $(240 \mathrm{mg} / 24 \mathrm{~h})$, intramuscular streptomycin (1 g/24 h), oral ciprofloxacin (500 $\mathrm{mg} / 12 \mathrm{~h}$ ), and co-trimoxazole (160/800/12 h). In neurobrucellosis patients, intravenous ceftriaxone $(2 \mathrm{~g} / 12 \mathrm{~h})$ was added to the regimen initially for 2-4 weeks. The treatment duration was 6-12 weeks in osteoarticular involvement, 12-24 weeks in neurobrucellosis, and 6-12 weeks for the other clinical forms.

The SPSS ver. 17.0 was used for the statistical analysis of data.

\section{RESULTS}

A total 192 patients were enrolled in this study, overall, the mean age of the patients included in the study was $36.16 \pm 11.21$ years (18-61 years), males outnumbered the females, $161(83.9 \%)$ vs $31(16.1 \%)$. Of these, 62 patients $(32.3 \%)$ were Indian, 21 (10.9\%) Egyptian, 54 (28.1\%) Bangladesh, 8 (4.2\%) Seri lanka, and 47 (24.5\%) Kuwaiti. Majority of the male patients were shepherds 144 (75\%) and veterinaries 12 (6\%), working in farms and had positive history of contact with the secretions of animals. The remaining of the patients $36(19 \%)$ consumed of unpasteurized dairy.

Fever (91.6\%), night sweats (85.9\%), arthralgia (76\%), and low back pain $(63.5 \%)$ were the most common presenting symptoms; (Table 1). The most common clinical findings were fever (91.6\%), peripheral arthritis (22.4\%), hepatomegaly (25\%), splenomegaly (21.3\%) and scrotal swelling (9.4\%), (Table 1).

C-reactive protein elevations (76.5\%), Aminotransferase elevations (28.1\%), Leukocytosis $(11.9 \%)$ and anemia $(9.98 \%)$ were the most frequent laboratory findings (Table 2). The Tubal agglutination test was positive in $100 \%$ of cases, with titers ranging from 1/160 to 1/2560. 106 patients revealed Brucella spp growth in their blood cultures (Table 2).

The focal involvement of infection occurred in about $42 \%$ percent of cases. The most common focal involvements were hepatic involvement (28.1\%), Osteoarticular involvement $(22.4 \%)$, Hematological involvement $(17.1 \%)$ and orchidepididymitis involvement $(9.4 \%)$. The other focal involvements are shown in Table (3).

The osteoarticular involvement included arthritis (22.4\%), sacroiliitis (14\%), spondylitis $(2.08 \%)$, and psoas abscess $(0.52 \%)$. The most common joints involvements were wrist joints, knee joints and hip joints. The commonest hematological 
abnormalities were leukocytosis (11.9\%), anemia (9.98\%), and thrombocytopenia (7.2\%) (Table 3).
The numbers of relapsing cases (21 cases) were $10.9 \%$ percent of cases.

Table (1) : Clinical manifestations of 192 patients with acute brucellosis

\begin{tabular}{|l|c|c|c|}
\hline \multicolumn{1}{|c|}{ Symptoms } & NO (\%) & Signs & No (\%) \\
\hline Fever & $176(91.6 \%)$ & Fever & $176(91.6 \%)$ \\
\hline Night sweat & $165(85.9 \%)$ & Splenomegaly & $41(21.35 \%)$ \\
\hline arthralgia & $146(76 \%)$ & Hepatomegaly & $48(25 \%)$ \\
\hline Fatigue & $102(53.1 \%)$ & Hepatoslenomegaly & $31(16.1 \%)$ \\
\hline Anorexia & $51(26.5 \%)$ & Lymphadenopathy & $11(5.7 \%)$ \\
\hline Weight loss & $41(21.3 \%)$ & Scrotal swelling & $18(9.4 \%)$ \\
\hline Myalgia & $75(39 \%)$ & Arthritis & $43(22.4 \%)$ \\
\hline Low back pain & $122(63.5 \%)$ & Heart murmur & $2(1.04 \%)$ \\
\hline Nausea/vomiting & $24(12.5 \%)$ & Neck stiffness & $1(0.52 \%)$ \\
\hline Headache & $28(14.5 \%)$ & Jaundice & $0(0.0 \%)$ \\
\hline Scrotal pain & $18(9.4 \%)$ & Rash & $0(0.0 \%)$ \\
\hline Cough & $9(4.6 \%)$ & & \\
\hline Shortness of breath & $2(1 \%)$ & & \\
\hline
\end{tabular}

Table (2) : Laboratory findings of 192 patients with acute brucellosis

\begin{tabular}{|l|c|}
\hline \multicolumn{1}{|c|}{ Laboratory findings } & NO (\%) \\
\hline ALT elevations $>5$ folds $(10-55 \mathrm{IU} / \mathrm{L})$ & $54(28.1 \%)$ \\
\hline AST elevations $>5$ folds $(5-34 \mathrm{IU} / \mathrm{L})$ & $54(28.1 \%)$ \\
\hline Total Bilirubin elevations $>20 \mathrm{umol} / \mathrm{L}$ & $0(0.0 \%)$ \\
\hline Serum creatinin elevations $>115 \mathrm{umol} / \mathrm{L}$ & $2(1.04 \%)$ \\
\hline Anemia $(\mathrm{HB}<140 \mathrm{~g} / \mathrm{L})$ & $19(9.98 \%)$ \\
\hline Leucopenia $(\mathrm{WBCs}<3600)$ & $16(8.3 \%)$ \\
\hline Leukocytosis $(\mathrm{WBCs}>11200)$ & $23(11.9 \%)$ \\
\hline Thrombocytopenia $(\mathrm{PLT}<150.000)$ & $14(7.2 \%)$ \\
\hline CRP elevation $>8 \mathrm{mg} / \mathrm{L}$ & $147(76.5 \%)$ \\
\hline ESR elevations $>20 \mathrm{~mm} / \mathrm{hr}$ & $34(17.7 \%)$ \\
\hline Rheumatoid factor positive & $0(0.0 \%)$ \\
\hline Tubal agglutination test $(\mathrm{TAT})>1: 160$ & $192(100 \%)$ \\
\hline Blood culture for brucella & $106(55.2 \%)$ \\
\hline
\end{tabular}

ALT: Alanine aminotransferase; AST: Aspartate Aminotransferase; CRP: C-reactive protein; ESR: erythrocyte sedimentation rate

Table-(3) : Localized infection (complications) of 192 patients with acute brucellosis

\begin{tabular}{|l|c|}
\hline \multicolumn{1}{|c|}{ Focal infection } & NO (\%) \\
\hline Hepatitis & $54(28.1 \%)$ \\
\hline Orchitis and epididymo-orchitis & $18(9.4 \%)$ \\
\hline Arthritis & $43(22.4 \%)$ \\
\hline Sacroiliitis & $27(14 \%)$ \\
\hline Spondylitis & $4(2.08 \%)$ \\
\hline Psoas abscess & $1(0.52 \%)$ \\
\hline Hematological abnormalities & $33(17.1 \%)$ \\
\hline CNS involvement (Neuro-brucellosis) & $1(0.52 \%)$ \\
\hline Cardiac involvement (endocarditis) & $2(1.04 \%)$ \\
\hline Renal involvement (glomerulonephritis) & $2(1.04 \%)$ \\
\hline
\end{tabular}




\section{DISCUSSION}

Brucellosis is considered a major medical problem in many countries worldwide where brucellosis is endemic. Brucellosis is endemic in Kuwait [2]. The epidemiology of brucellosis in Kuwait was changed over the last decades, reported infection rates decreased from 68.9 per 100,000 population in 1985 reaching the level 2.1 per 100,000 population in 2006 [6]. This is may be attributed to eradication programs among animals, socioeconomic changes and improved disease recognition.

Brucellosis can occur at any age but it is more prevalent in the adolescents and young adults [7]. In the current study, the age of patients ranged from 18 to 61 years and $76 \%$ of patients were between 18 and 44 years. Some studies reported that brucellosis is more frequently occurs in this age group [8] because this age group is the most productive group in the community and most of them working on farms with dairy production e.g shepherds and veterinaries. Brucellosis can occur in both genders [9]. In the present study, males outnumbered the females, $161(83.9 \%)$ vs 31 $(16.1 \%)$. Male were at higher risk of acquiring this disease than female because The male may be more likely to come into contact with infected animals and have more opportunities to drink raw milk than female. Also, in the current study, the prevalence of brucellosis is high among shepherds and veterinarians and this may be another reason why the prevalence is higher among males than females. This also leads to fact that brucellosis is considered an occupational disease.

Many routs have been implicated in the transmission of Brucellosis and its prevalence. In the endemic countries, the primary route of transmission is by the ingestion of raw or unpasteurized dairy products; whereas in the developed countries, infection can occur due to occupational exposure [10]. In the current study, the main routs of transmission were consumption of unpasteurized dairy products and contact with infected animals. The consumption of unpasteurized dairy products has been reported in 69 percent of cases in Kuwait by Mousa et al., [11]. Interhuman transmission of brucellosis has been reported in one female patient who probable got the disease from her husband by sexual contact. Similar to some reports that documented the sexual transmission of brucellosis [12].
Brucellosis is a systemic infection which can affect any system, tissue or organ of the body. Organ involvement can be considered as a complication or as focal involvement. The present study showed that $42 \%$ percent of cases had focal involvement or complications of brucellosis. Hepatic involvement is seen as the most frequent complication of brucellosis in this study (28.1\%) in the form of mild hepatitis and no cases were reported with acute liver failure. This is in accordance with T. Buzgan et al. [13] who detected 24.8 percent of the cases of brucellosis had Elevated transaminase levels. Rarely, Brucella can also cause splenic or hepatic abscesses, acute cholecystitis, colitis, pancreatitis and peritonitis [14] and none of these complications were recorded in this study.

Brucellosis is frequently associated with osteoarticular involvement [15]. Osteo-articular involvements include peripheral arthritis, sacroiliitis, spondylitis, Paravertebral abscess and psoas abscess [16]. Osteo-articular involvement occurs in 20-85\% of cases [17]. Osteo-articular involvement was noted in $39 \%$ of acute cases in the present study. Infection of the joints is the most common localized osteo-articular complication of brucellosis and a frequent cause of infectious arthritis in countries where brucella is endemic [15]. In the present study, Peripheral arthritis had been reported in 22.4 percent of cases affecting mainly wrist, knee, hip joints and sacroiliitis had been reported in 14 percent of cases. Four cases $(2.08 \%)$ of our 192 patients exhibited spondylitis. In accordance with Turan Buzgan et al. [13] we found that the lumbar spine was the most frequent site of spondylitis.

Genitourinary involvement has been noted in 2 to $10 \%$ of the patients with brucellosis and epididymoorchitis is the most frequent genitourinary complication. The incidence of epididymoorchitis in brucellosis is estimated at 2\%-20\% [18]. In the present study, epididymoorchitis occurred in $9.4 \%$ of all patients with brucellosis which is in accordance with Khan [19] who investigated 100 patients with brucellosis in Saudi Arabia and found testicular involvement in $6 \%$.

Hematological changes are frequent in brucellosis, but these hematological abnormalities are usually mild such as anemia, leukopenia and Thrombocytopenia [20] and serious hematological involvement is rare e.g Disseminated intravascular coagulation. In the current study, anemia was 
found in 19 patients (9.98\%), leukopenia in 16 $(8.3 \%)$, thrombocytopenia in $14(7.2 \%)$ and Disseminated intravascular coagulation was not reported. This figure is lower than reported by Kdeniz et al. [21] who found that anemia was present in 128 patients (55\%), leukopenia in 49 (21\%), thrombocytopenia in $59(26 \%)$ and pancytopenia in $18(8 \%)$ patients.

Neurobrucellosis has been reported in $2 \%$ to $5 \%$ of the patients with brucellosis [22]. Meningitis or meningo-encephalitis is the most frequent neurological complications of brucellosis [22]. In our study, only one case $(0.52 \%)$ was diagnosed with neurobrucellosis. This low incidence of CNS involvement in current study may be due to management of this especial group in general hospital and not refer to IDH.

In this study, two (1.04\%) brucellosis patients exhibit renal involvement. one patient presented with Glomerulonephritis and another with interstitial nephritis and endocarditis. in some case reports, Glomerulonephritis and tubule-interstitial nephritis have been reported [17].

Cardiovascular involvement of Brucellosis can result in endocarditis, myocarditis or pericarditis. Endocarditis is the most frequent presentation of cardiovascular involvement, which is documented in less than $2 \%$ of patients [13]. Endocarditis is a very severe complication of brucellosis. The aortic valve has been commonly involved, followed by the mitral valve alone or both valves concurrently. The aortic valve is affected in $75 \%$ of patients, and $50 \%$ of affected valves were previously healthy [22]. In the present study, endocarditis was found in 2 patients (1.04\%) with aortic valve involvement. The valve replacement was done for the two patients.

In the present study, the small sample size of the studied patients and only one center where the study was conducted have limited the power to detect and describe all the clinical presentation, laboratory findings and complications of brucellosis.

\section{CONCLUSION}

Brucellosis is an endemic disease in Kuwait and considered an important health problem and it has a significant morbidity. The consumption of raw dairy products as well as occupational risks of contact with infected animal has been reported the main rout of transmission the disease in.
Additionally, the disease affects primarily persons in their productive age.

Diagnosis of the disease is not difficult because it based on epidemiologic data, clinical manifestations, laboratory investigations and high degree of suspicion by the physicians. But the diagnosis of localized forms of brucellosis can occasionally be difficult because of misdiagnosis with other diseases.

There is no recommended protocol for management of complicated brucellosis. Further studies are needed to assess the most appropriate treatment choices and durations in complicated brucellosis. Eradication of the disease in humans can be done by the public health education and the control of the disease in animals.

Funding: None.

Conflicts of interest: None.

Ethical approval:Approved .

\section{REFERENCES}

1- Kassiri H, Amani H, Lotfi M. Epidemiological, laboratory, diagnostic and public health aspects of human brucellosis in western Iran. Asian Pac J Trop Biomed, 2013; 3(8):589-94.

2- Pappas G, Papadimitriou P, Akritidis N, Christou $\mathrm{L}$, Tsianos EV. The new global map of human brucellosis. Lancet Infect Dis, 2006; 6:91-9.

3- Hendaus MA, Qaqish RM, Alhammadi AH. Neurobrucellosis in children. Asian Pac J Trop Biomed, 2015; 5(2):158-61.

4- Roushan MR, Amiri MJ. Update on childhood brucellosis. Recent Pat Antiinfect Drug Discov, 2013;8(1):42-6.

5- Hasanjani Roushan MR, Mohrez M, Smailnejad Gangi SM, Soleimani Amiri MJ, Hajiahmadi M. Epidemiological features and clinical manifestations in 469 adult patients with brucellosis in Babol, Northern Iran. Epidemiol Infect, 2004; 132(6): 1109-14.

6- Ministry of Health (MOH), Kuwait, Department of public health. Infectious Diseases Annual Report, 2006.

7- Zeinalian Dastjerdi M, Fadaei Nobari R, Ramazanpour J. Epidemiological features of human brucellosis in central Iran, 2006-2011. Public Health, 2012; 126(12):1058-62.

8- Savas L, Onlen Y, Savas N, Yapar AF, Aydin M, Tugal O. Prospective evaluation of 140 patients with brucellosis in the southern region of Turkey. Infect Dis Clin Pract, 2007; 15(2):83-8.

9- Jama'ayah MZ, Heu JY, Norazah A. Seroprevalance of brucellosis among suspected cases in Malaysia. Malays J Pathol, 2011; 33(1):31-4. 
10- Alavi SM, Motlagh ME. A review of epidemiology, diagnosis and management of brucellosis for general physicians working in the Iranian health network. Jundishapur J Microbiol, 2012; 5(2): 384-7.

11- Mousa AR, Elhag KM, Khagali M, Marafie AA. A nature of human brucellosis inKuwait: study of 379 cases. Rev Infect Dis, 1988; 10:211-7.

12- Meltzer E, Sidi Y, Smolen G, Banai M, Bardenstein S, Schwartz E. Sexually Transmitted Brucellosis in Humans. Clinical Infectious Diseases, 2010; 51(2): e12-e15.

13- Buzgan T, Karahocagil MK, Irmak H, Baran AI, Karsen H, Omer Evirgen O, et al. Clinical manifestations and complications in 1028 cases of brucellosis: a retrospective evaluation and review of the literature. International Journal of Infectious Diseases, 2010; 14: e469-e478.

14- Young EJ. Brucella species. In: Mandell GL, Bennet JE, Dolin R, editors. Principles and Practice of Infectious Diseases. 5th ed.Philadelphia: Churchill Livingstone, 2000; pp. 2386-2393.

15- Geyik MF, Gur A, Nas K, Cevik R, Sarac J, Dikici B, et al. Musculoskeletal involvement of brucellosis in different age groups: a study of 195 cases. Swiss Med Wkly, 2002; 132(7-8):98-105.
16- Gonzalez-Gay MA, Garcia-Porrua C, Ibanez D, Garcia-Pais MJ. Osteoarticular complications of brucellosis in an Atlantic area of Spain. $J$ Rheumatol, 1999; 26: 141-145.

17- Young EJ. Brucella species. In: Mandell GL, Bennett JE, Dolin R, editors. Principles and practice of infectious diseases. 6th ed., Philadelphia: Churchill Livingstone, 2005; p. 2669-72.

18- Navarro-Martinez A, Solera J, Corredoira J, Beato JL, Martinez-Alfaro E, Atienzar M et al. Epididymoorchitis due to Brucella melitensis: a retrospective study of 59 patients. Clin Infect Dis, 2001; 33: 2017-2022.

19- Khan MY. Brucellosis: observations on 100 patients. Ann Saudi Med, 1986; 6: 519-523.

20- Ayasha HM, Shayib MA. Pancytopenia and other hematological findings in brucellosis. Scand $J$ Haematol, 1986; 36: 335-338.

21- Akdeniz H, Irmak H, Seckinli T, Buzgan T, Demiroz AP. Hematological manifestations in brucellosis cases in Turkey. Acta Med Okayama, 1998; 52: 63-65.

22- ERTEK M, YAZGI H, KADANALI A, ÖZDEN K, TAfiYARAN MA. Complications of Brucella Infection among Adults: An 18-Year Retrospective Evaluation. Turk J Med Sci, 2006; 36 (6): 377 381. 